COMMISSION No. 21

\title{
LIGHT OF THE NIGHT SKY (LUMIERE DU CIEL NOCTURNE)
}

\author{
Report of Meetings 3,4,8 and 10 August 1988
}

PRESIDENT: K. Mattila

SECRETARY: A.C. Levasseur-Regourd

\section{August 1988}

\section{BUSINESS SESSION}

The President welcomed with pleasure the large number of members attending the Commission 21 sessions in Baltimore.

\section{COMMISSION MEMBERSHIP AND OFFICERS}

The President reported the sad news about the death of two members of the Commission, Dr. Carlos Sánchez-Magro and Professor Richard H. Giese, and reminded briefly on their scientific work related to Commission 21. Prof. Giese was a central and beloved figure in Commission 21 for many years. He served in the Organizing Committee since 1973 and as President of Commission 21 during the triennium 1982-1985. The president invited the participants to stand up in silence in memory of these two outstanding scientists and friendly colleagues. (Obituaries have been published in Commission 21 Newsletters Nos. 9 and 11).

Concerning the officers and membership the following list was approved:

President: A.C. Levasseur-Regourd

Vice-President: M.S. Hanner

Organizing Committee: S. Bowyer, R. Dumont, Yu.I. Galperin, J. Houck, Ph. Lamy, Ch. Leinert, K. Mattila, T. Mukai

New Members: J. Clairemidi, L.B. d'Hendercourt, S.F. Dermott, A. Fujiwara, H. Hasegawa, M.G. Hauser, A. Leger, K. Lumme, T. Maihara, J.-M. Perrin, G. Toller, K. Yamashi, T. Yamamoto

Consultants 1988-91: C. Classen, M. Dubin, G. Eichorn, A. Frey, M. Ilyas, D.J. Kessler,

B. Kneissel, W. Kokott, G. Lopez, J. Maucherat, J.A.M. McDonnell, R.D. Mercer, J. Michael, A. Mujica, A.W. Peterson, H. Radoski, E.T. Rusk, R. Schaefer, G.H. Schwem, W.-X. Wang, K. Weiss-Wrana, H.A. Zook

\section{REPORTS ON ASTRONOMY 1988}

The President thanked the members for sending information on their activities in 1985-88 and acknowledged the help provided especially by A.C. Levasseur-Regourd in the preparation of the report of the Commission.

\section{THE NEWSLETTER OF THE COMMISSION}

The President edited three newsletters, Nos. 9, 10 and 11 during this triennium.

\section{OTHER COMMISSION ACTIVITIES IN 1985-88}

The President, on behalf of the Commission, has expressed, in a letter addressed to the General Secretary, his concern about the increasing threat of light pollution especially through the planned commercial uses of near - Earth environment, e.g. the French Eiffel Tower in Space and the US Celestis \& Space Services Inc. projects. Commission 21 is co-sponsor of the IAU Colloquium No. 112 "Light Pollution, Radio Interference and Space Debris", 13-16 August 1988.

Commission 21 co-sponsored the IAU symposium No. 135 "Interstellar Dust", 26-30 July 1988. It was the main sponsor of the Joint Discussion IV on August 51988 during the GA in Baltimore on "The Cosmic Dust Connection in Interplanetary Space: Comets, Interstellar Dust and Families of Minor Planets"; it was co-sponsored by Commissions 7, 15, 20, 22, 34 and 49. 


\section{FORTHCOMING ACTIVITIES}

The President described the preparations under way for IAU Symposium No. 139 "Galactic and Extragalactic Background Radiation - Optical, Ultraviolet and Infrared Components", to be arranged on 12-16 June 1989 in Heidelberg, FRG. The organization of a meeting on this topic has been discussed and planned during many Commission 21 sessions in the past. Therefore it is natural that Commission 21 is the main sponsor of the Symposium, which is being co-sponsored by IAU Commissions 28, 33, 34, 44 and 47 and by COSPAR Subcommission E.1. The Chairman of the Scientific Organizing Committee is K. Mattila, and the Chairman of the Local Organizing Committee is Ch. Leinert. The host institute is the Max-Planck-Institut für Astronomie.

Dr. T. Mukai described the preparations for the proposed IAU Colloquium (now approved as IAU Coll. No. 126 by the EC) "Origin and Evolution of Interplanetary Dust", which is to be held on 27-30 August 1990 in Kyoto, Japan. This meeting will continue the series of highly successful meetings on interplanetary dust, the first of which was in 1967 in Honolulu, followed by the meetings in Heidelberg (1975), Ottawa (1979) and Marseille (1984). Commission 21 is the main sponsor and Commissions 15 and 22 as well as COSPAR are the co-sponsors of this colloquium. The chairperson of the SOC is A.C. Levasseur-Regourd and the Local Organizing Committee is chaired by $\mathrm{H}$. Hasegawa.

\section{WORKING GROUPS}

"Working Party" on Interplanetary Pollution: Commission 21 supported the establishment by Comm. 22 of a Working Party with the task to recommend an IAU/COSPAR Panel on Interplanetary Pollution. Suitable Commission 21 representatives for the Working Party will be nominated later by the Organizing committee.

Working Group on Fluffy Structures : On the initiative of J.M. Greenberg Commission 21 decided to set up a WG on this topic. The Chairman is J.M: Greenberg, and the other members T. Mukai, R. Zerull and W.-X. Wang.

Working Group on "Standard Tables of the Light of the Night Sky". This WG is described in the report of the scientific session on 8 August 1988.

\section{CLOSING REMARKS}

The President expressed his thanks to the members and to the Organizing Committee of Commission 21 for their cooperation. He thanked especially the two resigning members of the OC, Dr. Tanabe and Dr. J. Weinberg for their long and valuable work in the Organizing Committee and as Commission Presidents. The incoming President expressed the members' thanks to the retiring President.

\section{SCIENTIFIC SESSIONS}

\section{August 1988}

\section{THREE-DIMENSIONAL MODELLING AND INVERSION TECHNIQUES RELATED TO THE ZODIACAL AND GALACTIC LIGHT \\ (Chair: A.C. Levasseur-Regourd)}

This session was devoted to the memory of Richard H. Giese. J. Weinberg presented some personal memories of Richard $\mathrm{H}$. Giese. The following papers were presented during the session, in order of their presentation:

W.B. Burton: Modelling the galactic infrared emission (invited review) - no abstract available 
B. Kneissel: The Three-Dimensional Distribution of Zodiacal Dust and its Dynamical Structure (invited review) - Global models of the 3-D distribution in the zodiacal dust cloud taken from visual observations prefer large number densities above the solar poles (classical fan, sombreros and bulge fan). But also multilobe structures are found. Infrared observations mostly demand for density nodes above the solar poles. Despite of this apparent divergences, many models give equal densities close to the Earth. The brightness given by each model has been evaluated with use of a scattering function similar to that for Allende-meteorites and compared to the brightness data of Levasseur-Regourd and Dumont. The convergence of models close to the Earth is confirmed. Dynamical analysis reveals that there is a least number condition for the helioecliptic dependent part of the density function, which is not satisfied by the sinks of multilobes. Furthermore the number of orbits with particles in retrograde motion according to the optimum sombreros exceeds by far the number given by other observations.

J.C. Good: Modelling of Zodiacal Infrared Emisstion (invited) - A physical model for the interplanetary dust cloud has been fit to the IRAS data. This model consists of spatial distributions for the dust volumetric emissivity and temperature, an inclination and line of nodes for the cloud, and a simple parametrization of the dust emissivity as a function of wavelength. The volumetric emissivity was found to vary as $r^{-1.8}\left[-5\left(\sin \beta_{n}\right)^{-1.3}\right]$ and the temperature as $r^{-0.35}$. The IRAS data are limited to solar elongation angles between $60^{\circ}$ and $120^{\circ}$ and consequently are not sensitive to material closer to the Sun than $0.9 \mathrm{AU}$. However, comparison of the predicted model flux and the AFGL Zodiacal Infrared Project (ZIP) data (which looked to within $22^{\circ}$ of the Sun) at 10 and $20 \mu \mathrm{m}$ shows excellent agreement and implies that the $\mathrm{r}^{-1.8}$ power law is good to $0.4 \mathrm{AU}$. The inclination and line of nodes are $1.7^{\circ}$ and $69^{\circ}$ respectively.

\section{A.C. Levasseur-Regourd and R. Dumont: Out-of-Ecliptic Nodes of Uncertainly Method,}

Inversion Results (invited) - Both zodiacal light and thermal emission measurements provide integrals along a line of sight of local brightnesses. Various attempts have been made towards an inversion of the integral in the ecliptic plane. The nodes of lesser uncertainty method is now extended to the out-of-ecliptic case. When coherent sets of measurements performed towards the ecliptic pole and in the ecliptic are available, the local brightness $J(\beta)$ along the Earth-ecliptic pole line is found to obey to 5 constraints. If all the possible $J(\beta o)$ functions are assumed to be monotonous, then they have to constrict in a nodal region, where they can be determined with less uncertainty than elsewhere. Typically, a node of lesser uncertainty is found for $\beta o \approx 20^{\circ}$, i.e. for a distance above the ecliptic $\approx 0.36$ AU or a distance to the $S u n \approx 1.07 \mathrm{AU}$. From this method, the local polarization is found to decrease with increasing $\beta$ o for a given distance to the Sun, while the local bulk albedo may simultaneously increase. These results are to be compared with the in-ecliptic variation with heliocentric distance of local polarization and albedo.

S.S. Hong and S.M. Kwon: Connection Between the Infrared Zodiacal Emission and the Visible Zodiacal Light (invited) - By inverting the brightness distribution of the zodiacal thermal emission along the ecliptic plane, we have examined how the volume absorption cross section $n(r) \sigma_{a b s}(r ; \lambda)$ of the interplanetary dust particles varies with the heliocentric distance $r$ for infrared wavelengths. The usual inverse power law of $\mathrm{r}$ is found to be inadequate for describing the spatial distribution of

$\mathrm{n}(\mathrm{r}) \sigma_{\mathrm{abs}}(\mathrm{r} ; \lambda)$ in the infrared. The resulting $\mathrm{n}(\mathrm{r}) \sigma_{\mathrm{abs}}(\mathrm{r} ; \lambda) \mathrm{s}$ at $\lambda=10.87 \mu \mathrm{m}$ and $\lambda=20.87 \mu \mathrm{m}$ decrease with $r$ less steeply than the $r^{-1.3}$ relation, which was known for the spatial distribution of the volume scattering cross section, $n(r) \sigma_{s c a}(r)$, of the dust in the visible. It is further found that the ratio of $n(r) \sigma_{a b s}(r ; \lambda)$ 's at the two IR wavelengths varies with $r$. This is a strong indication that the zodiacal dust cloud contains more than one dust species and their mixing ratios vary with $r$. Comparison of $n(r) \sigma_{a b s}(r ; \lambda)$ in the infrared with $n(r) \sigma_{\text {sca }}(r)$ in the visible suggests that the $r^{-1.3}$ relation is too steep a decline for the spatial distribution of the dust number density $n(r)$ in the ecliptic plane.

M.G. Hauser and J.M. Vrtilek: IRAS Measurements of the Solar Elongation Dependence of the Zodiacal Emission and the Inversion of the Infrared Brightness Integral - We examine the infrared brightness of interplanetary dust in the ecliptic and its variation with solar elongation angle, using IRAS data in the 12,25 , and 60 micrometer bands. Over the entire elongation range covered ( 60 to 120 degrees), a quadratic function is an excellent fit to the peak brightness of the zodiacal 
emission as a function of solar elongation in these three IRAS bands. Using our function fits to the elongation-dependent brightness, we have applied an inversion technique related to that of Hong and Um (1987, Astrophys. J., Vol. 320, 928) to the problem of determining the value at 1 A.U. and the heliocentric distance dependence of the volumetric absorption cross-section of the interplanetary dust. We will discuss the results of the inversion approach and their sensitivity to several key assumptions.

S.F. Dermott, P.D. Nicholson, R.S. Gomes and R. Malhotra: Modelling of the IRAS Solar System Bands (invited) - Shortly after the discovery of the solar dust bands by the IRAS spacecraft it was suggested that the bands that extend uniformly round the sky arise from the gradual comminution of asteroids in the most prominent Hirayama asteroid families (Dermott et al.: Nature 312, 505-509 (1984)). Stringent observational tests have been devised to test this hypothesis. These tests are robust in that they are geometrical in nature and can be made with observations in a single waveband. Thus, they exploit the high resolution and pointing stability of the IRAS spacecraft, while side-stepping the problem of absolute calibration. Rigorous simulation of the observational data has been achieved by a numerical model that allows us to calculate, for any distribution of particle orbits in the solar system, the brightness scans that would have been seen by IRAS in any one observing period at any elongation angle. If we allow (and this is essential) that the orbits of the particles have decayed due to Poynting-Robertson light drag and that the orbital elements of the particles are now dispersed about those of the asteroidal family members, then the observations that we have analysed todate appear to be in close agreement with the predictions of the Hirayama family model.

S. Mukai and T, Mukai: Backscattering by a Large Particle with Rough Surface - A similarity of backscattering features (negative polarization and backward enhancement of intensity) exist among the scattering lights by Moon, asteroid, cometary dust and interplanetary dust. We show that multiple reflections by a micro-structured rough surface including shadowing effect cause the above features. Application to P/Halley gives a significant fit to the observed results.

\section{$\underline{8 \text { August } 1988}$}

\section{STANDARD TABLES OF THE COMPONENTS OF THE LIGHT OF THE NIGHT SKY} (Chair: J. Weinberg, Secretary: G. Toller)

Some of the considerations which had motivated the Organizing Committee to arrange this session were the following: Several of the IAU Commissions have used their expertise to put together recommendations for some astronomical constants, names etc. in their field. We would like to ask ourselves and discuss whether we could (and should) try to combine the existing knowledge on the different components of the light of the night sky into something like the "Standard tables for the LONS". Such tables could be used e.g. by astronomers from other fields who need to know the sky brightness at a given time and a given position on the sky. These considerations have been related so far mainly to the optical domain but are certainly also timely for the infrared.

The following papers were presented at the session, in order of their presentation:

A.C. Levasseur-Regourd and R. Dumont: Available tables for zodiacal light intensity distribution (invited review) - Reliable evaluations of zodiacal light intensity, polarization or emission should be available from any point of observation, at any wavelength, at any time, in any viewing direction, not only to obtain a better knowledge of the interplanetary dust complex, but also to obtain an accurate estimation of the zodiacal "foreground". Such a foreground is indeed most significant for HST, IRAS, COBE, ISO, etc., observations. A review of various tables, as given during the last two decades by Smith et al., Dumont, Frey et al., Dumont and Sanchez, Classen, Torr et al., Levasseur-Regourd and Dumont, Pfleiderer and Leuprecht, etc., is presented. The problems of the wavelength dependence (solarlike for zodiacal light) and the time dependence (no significant correlation with solar activity) have been solved. However, the tables may need to be corrected for 
some small scale local enhancements (dust trails,...) and for the changing position of the observer with respect to the warped symmetry surface of the zodiacal cloud.

M.G. Hauser: Distribution of the Infrared Zodiacal Emission (IRAS) (Invited review) - The data from the IRAS survey show that the large-scale brightness of most of the sky at wavelengths from $12 \mu \mathrm{m}$ to $100 \mu \mathrm{m}$ is dominated by thermal emission from interplanetary dust. A variety of techniques have been utilized to segregate and model this 'zodiacal emission'. Analyses of the annual variation of ecliptic polar brightness and latitude of peak brightness provide evidence of a tilted, warped symmetry surface of the dust cloud. Available representations of the zodiacal emission now include averages of IRAS scan data, empirical fits to the scan data, and parameterized physical models. a new version of the IRAS data with improved calibration will be released in 1988. NASA's COBE mission will extend the wavelength coverage and photometric quality of zodiacal emission measurements.

J. Weinberg: Standard Tables of the Background Starlight Distribution (invited review) - A brief discussion was presented on post-war efforts to provide observational data on the background starlight. Examples of background starlight derived from Pioneer 10 and from Helios A were used to illustrate some of the more recent (space) data that are now available, precautions that should be taken in their use, and general considerations required in any effort to provide such a "standard" data set.

T.N. Gautier: Distribution of the Galactic Infrared Background Starlight (invited review) - no abstract available

H. Tanabe: Report on star counts at the Tokyo astronomical observatory - Since 1968, star counts on the Palomar Sky Survey Atlas have been made by using a then newly designed instrument in order to estimate background brightness in zodiacal light and airglow observations. As the results, blue and red brightnesses of the integrated starlight are presented for 24 sky regions (total measured area: 54.73 sq.deg.) including 4 polar (NCP, NEP, NGP and SGP) and 10 ecliptic regions.

These presentations were followed by a general open discussion with the title "Standard Tables: Why, How, When and Who?" Some of the considerations coming up in this discussion were

Units: Papers should supply conversion factors to energy units, i.e. both to $I_{\lambda}\left[\mathrm{erg} \mathrm{cm}^{-2} \mathrm{~s}^{-1}\right.$ sterad $^{-1}$ $\left.\AA^{-1}\right]$ and to $I_{v}\left[\mathrm{erg} \mathrm{cm}^{-2} \mathrm{~s}^{-1}\right.$ sterad $\left.{ }^{-1} \mathrm{~Hz}^{-1}\right]$. The frequently used night-sky unit $S_{10}(\mathrm{~V})_{\mathrm{G} 2 \mathrm{~V}}$ is acceptable for zodiacal light photometry but conversions should be supplied. In the photometry of the galactic and extragalactic components $S_{10}$ unit is mostly not referred to the solar spectrum. Also the bandpasses of the filters used in the photometry should be published. A table of standard conversion factors used in the night sky photometry should be published in a widely distributed reference work, such as Allen: Astrophysical Quantities.

Caution in use of "Standard Tables": When using zodiacal light tables one should be aware of the time-dependence of the brightness distribution due to the changing position of the observer relative to the symmetry surface of the zodiacal cloud. Caution was urged in the use of the term "standard", since there is currently no single standard or "best" data set available for any of the components of the light of the night sky (LONS). It was urged that more detail be provided on LONS reduction methodology, calibration, and units when reporting such data.

Working Group on "Standard Tables" : It was decided to form a Working Group within Commission 21 which will have the task to review the existing light of the night sky tables, and provide the "best" compilations for publication in a widely distributed reference book, such as Allen: Astrophysical Quantities. It was agreed that the members of the WG should represent the optical, IR and UV domains and equally well the different LONS components, zodiacal light, background starlight and star counts. The following persons were elected to the WG: A.C. Levasseur-Regourd 
(Convener), S. Bowyer, T.N. Gautier, M.G. Hauser, J. Houck, C. Lillie, H. Tanabe and J. Weinberg, and it was agreed that the WG can invite new members.

\section{August 1988}

OPTICAL, IR AND UV LIGHT OF THE NIGHT SKY -- RECENT OBSERVATIONAL TOPICS AND FUTURE PLANS

(Chair: K. Mattila, M.S. Hanner)

The following papers were presented during the session, in order of their presentation:

\section{a. Galactic and Extragalactic Components}

\section{F. Low: Infrared Cirrus (invited review) - no abstract available}

T. Matsumoto: Infrared Cosmic Background Radiation (invited review) - Diffuse sky brightness was observed both in near-infrared and submillimeter regions with rocket-borne radiometers cooled by liq. $\mathrm{N}_{2}$ and He, respectively. Around $2 \mu \mathrm{m}$, a considerable amount of isotropic emission was observed which can possibly be attributed to the extragalactic origin. In submillimeter region, besides excess brightness over $2.74 \mathrm{~K} \mathrm{CBR}$, a significant isotropic emission at $137 \mu \mathrm{m}$ was detected. However, its origin and nature is not certain.

C. Heiles and W.T. Reach: Separating the Gaiactic and Zodiacal IR Emission (invited) - We are developing a physical model of the Zodiacal dust consisting of two components, a finite disk and rings that produce the 'Zodiacal bands'. We determine the location and shape of the components from the variation of their distances with solar elongation and time of year, a kind of 'parallax'. The method involves least-square fits to parameterized models and includes Gaussians to represent the 'bands' and a term in Galactic HI to represent the Galactic emission. We have determined the local emissivity, the orbital elements of the planes of the Zodiacal disk and rings, and the height of the rings above their mean plane, all to high accuracy.

S. Bowyer: Galactic and Extragalactic UV Background Radiation (invited review) - Our knowledge of the night sky background in the 100-4000 $\AA$ band was reviewed. Sources of diffuse emission in the band include resonantly scattered solar radiation from HeII in the Geocorona $(304 \AA)$ and from the local interstellar medium flowing through the heliosphere (HeI $584 \AA$ and HI $1216 \AA$ ). The local hot interstellar medium will produce line emission in the 100-912 $\AA$ band; at longer wavelengths sources of diffuse emission include hot gas in the Galaxy, scattering by dust, zodiacal light and possibly an extragalactic component.

Although our knowledge of most of these compnents is extremely limited, ongoing studies of the resonantly scattered hydrogen and helium in the heliosphere have led to an excellent characterization of the local interstellar medium. Recent studies of other components have identified emission from a hot $\left(10^{5} \mathrm{~K}\right)$ gas associated with a dynamic galactic halo. An extragalactic component has been identified associated with the UV output of distant galaxies and the intensity of the radiation puts severe constraints on the star formation rate over the last one third of a Hubble time. Finally, studies of scattered light have yielded a characterization of UV scattering dust grains.

R.C. Henry, J. Murthy and P.D. Feldman: UVX Observations of the Cosmic Ultraviolet Background - Observations of the cosmic background light in the wavelength range $1250 \AA$ were made with the Johns Hopkins UVX experiment flown on the Space Shuttle Columbia in January 1986. Eight targets, at galactic latitudes from $10^{\circ}$ to $90^{\circ}$ were observed. It is found that the cosmic background is patchy, ranging in birghtness from 300 to 900 photons $\left(\mathrm{cm}^{2} \mathrm{~s} \mathrm{sr} \AA\right)^{-1}$, with no correlation between hydrogen column density and cosmic background brightness.

J. Murthy: Probing the Infrared Cirrus with Ultraviolet Radiation - We are using the IRAS data in an effort to probe the global distribution of interstellar dust in the galaxy. Our method is to search 
the sky survey plates for enhancements of the cirrus near hot, UV-emitting stars, which dominate the ISRF up to several parsecs from the star. In a preliminary search of 25 hot stars, we have found such enhancements near five of them, implying that the filling factor of the dust is $25 \%$. This work is continuing in more detail.

R.A. Kimble, R.C. Henry and F. Paresce: The Hopkins Ultraviolet Background Explorer -- We describe the scientific goals and conceptual design of a proposed satellite mission for studying the far ultraviolet background in the wavelength range from 1230 to $1800 \AA$. By performing a complete sky survey as well as deep pointings with both a broadband camera and imaging long-slit spectrograph, we hope to investigate a variety of diffuse emission processes arising in the interstellar medium of our own galaxy and possibly in the intergalactic medium as well.

K. Mattila: Prospects for Measuring the Extragalactic Background in the Optical — There have been several attempts to measure the EBL but no generally accepted values are available so far. Because the EBL is expected to be only a minor fraction of the total night sky brightness a direct separation technique is entangled with great difficulties (see e.g. Dube, Wickes and Wilkinson, Ap.J. 232, 333, 1979). In the talk a new measurement of the EBL by Mattila and Schnur was presented in the area of the dark nebula L1642 in the anticentre direction. The method is basically the same as used by Mattila (Astron. Astrophys. 47, 77, 1976) in the L134 observations, but the observing and data analysis methods have been cosiderably improved.

\section{b. Solar System and Atmospheric Components}

A.C. Levasseur-Regourd and R. Dumont: Interplanetary Dust: Post-IRAS View (invited review) - Thermal emission from the interplanetary dust cloud has been confirmed by IRAS to be the most prominent component of the sky brightness in the 10-25 $\mu \mathrm{m}$ range. It is indeed a severe foreground for astronomical observations. It is also a major source of information on the local properties of the dust grains. Unlike the solar scattered light, the thermal emission of the grains is isotropic; even more progress can therefore be expected from the inversion of IRAS integrated birghtnesses.

The pre-IRAS understanding of the interplanetary dust cloud is reviewed. Recent results obtained through inversion methods are presented. In the ecliptic near $1 \mathrm{AU}$, the heliocentric gradient of temperature is about -0.33 , i.e. conflicts with a greybody assumption, while the gradient of albedo is about -0.7 , i.e. conflicts with a homogeneous cloud assumption. Out of the ecliptic, the dust distribution is still open to dispute. It is however neither smooth (dust bands and trails, warped symmetry surface), nor homogeneous (location dependence of local polarization or albedo). Such results imply that the mixing ratios of the various interplanetary dust sources (comets, asteroids, $\beta$ meteoroids) strongly depend upon location in the cloud.

P. Temi. P. De Bernardis, S. Masi, G. Moreno, A. Salama: Balloon Observations of the Zodiacal Thermal Emission - Results from an IR sky survey performed at wavelengths $11,19,50,108$ and $225 \mu \mathrm{m}$ during a balloon flight (ARGO) on July 30, 1984 are presented. A new physical model of the interplanetary dust thermal emission is then discussed. Based on very simple assumptions (blackbody, silicate or graphite grains, spherical and homogeneous), the model agrees with all available observations of the dust IR emission (AFGL, ZIP, ARGO, IRAS). The decrease of the volumetric absorption cross section with increasing heliocentric distance is found to follow a power law with exponent $\sim-1$.

J.M. Vrtilek and M.G. Hauser: IRAS measurements of diffuse solar system radiation - Using IRAS data, we present an overview of the properties of infrared emission from the interplanetary dust cloud. To assist in separating the smooth component of the interplanetary emission from Galactic emission, discrete sources, and the zodiacal bands, and to characterize the properties of the interplanetary emission in compact from, we introduce an empirical function whose adjustable parameters have simple geometric interpretations. This function is fitted in a lower envelope sense to individual IRAS scans; the function represents the data well, with rms residuals in the 25 micrometer band of only $0.3 \mathrm{MJy} / \mathrm{sr}$ (less than one half of one percent of the peak emission in this band). We 
take advantage of the nearly $1 \mathrm{yr}$ duration of the IRAS observations to study the time variation of the ecliptic latitude of peak brightness in the zodiacal emission, obtaining an inclination to the ecliptic $i=1.6 \pm 0.1^{\circ}$ and a line of ascending nodes $\Omega=41.1 \pm 0.6^{\circ}$ ( 1 sigma) for the symmetry surface of the interplanetary dust cloud. Disagreement in $\Omega$ between our measurements and previous determinations that sample different parts of the dust cloud supports the suggestion that the cloud lacks a true symmetry plane.

M.V. Sykes: Infrared Emission from Zodiacal Structures Arising from Asteroidal Collisions (invited) - The zodiacal dust bands arise from asteroid collisions. Several are now associated with the major Hirayama asteroid families, which were formed by the catastrophic breakup of larger asteroids some time in the past. Not all asteroid families, however, have dust bands, and not all dust bands are associated with asteroid families. This suggests that some bands may be the products of the present ( $\leq 10$ million years) collisional disruption of smaller asteroids. Four bands have now been resolved within a few degrees of the ecliptic - one pair associated with the Themis family, the other with the Koronis family. Comparison of dust band models with the observations indicate that the dust band particles have the same mean orbital elements as their associated asteroid family members, but have a greater dispersion in those elements. The dust band particles are found not to have experienced significant orbital decay by Poynting-Robertson drag, indicating that their lifetimes are collision dominated. Dust must eventually spiral in under radiation forces, but their surface brightness relative to the source region is small. It is predicted that long-duration spacebased dust collection experiments will detect a four-peak modulation in the interplanetary dust flux as a consequence of dust originating in the Koronis and Themis families.

S. Koutchmy: The Inner Zodiacal Dust Cloud (invited) - The analysis of the inner zodiacal cloud is still in a state of infancy due to the lack of a proper method for observing the faint halo of the F-corona around the solar disc. Total solar eclipses offer a rare and short opportunity to perform polarimetric, doppler shift and thermal emission measurements which are much needed. A weak evidence of an IR signature of olivine particles of 100 microns size has been presented by Mizutani et al. thanks to their balloon experiment flown during the 1983 eclipse. Spaceborne experiments have collected few exciting observations in white-light. Interplanetary travelling clouds are seen on the zodiacal cloud background monitored with the Helios Spacecraft photometers (B. Jackson) and the sudden disappearance of several sun-grazing comets were observed with the P-78 NRL coronagraph, producing a huge cloud of evaporating particles in the solar neighbourhood (D. Michels et al.). Many results are now anticipated in the frame of the SOHO mission with several coronagraphs.

P.D. Feldman, J. Murthy and R.C. Henry: UVX Observations of Ultraviolet Zodiacal Light Emission - Observations of the zodiacal light in the wavelength range 2000-3100 $\AA$ were made with the Johns Hopkins UVX experiment flown on the Space Shuttle Columbia in January 1986. Eight targets, at elongations from 101 to $133^{\circ}$ were observed. It is found that the uv color (relative to the visible) increases nearly linearly with ecliptic latitude implying that the small grains responsible for the uv scattering have a larger scale height than do the larger grains responsible for the visible scattering.

P.V. Kulkarni: Night Time All-Sky Temperature Measurement at the F-Region Height in the Earth's Atmosphere - A new instrument pointing to the zenith with a fish eye lens, prefilter, Fabry-Perot Spectrometer and an image intensifier is described to photograph, several times during a night, the temperature broadened interference fringes from the dome of sky emitting [OI] $6300 \AA$. When fringes are radially scanned temperatures at several points in the sky would be obtained simultaneously. A design of the system is given.

R.H. Garstang: Modelling the Night Sky Glow from Cities - The author described his continuing calculations of the contribution of man-made light pollution to night sky brightness. His model has been improved by the inclusion of earth curvature and by extension to B magnitudes. Applications have been made to may U.S. Observatories and possible observatory sites. Population projections from federal and state agencies are being used to predict the future growth of light pollution. 